\title{
A MASSA GORDA DE RISCO AFETA A CAPACIDADE AERÓBIA DE JOVENS ADOLESCENTES
}

\author{
THE RISK FAT MASS CLASS AFFECTS AEROBIC CAPACITY OF YOUNG ADOLESCENTS \\ LA MASA DE TEJIDO ADIPOSO DE RIESGO AFECTA LA CAPACIDAD AERÓBICA \\ DE JÓVENES ADOLESCENTES
}

Luís Massuça (Educador Físico) $)^{1,2}$ Jorge Proença (Educador Físico) ${ }^{1}$

1. Faculdade de Educação Física e Desporto, Universidade Lusófona de Humanidades e Tecnologias, Lisboa, Portugal.

2. ICPOL, Instituto Superior de Ciências Policiais e Segurança Interna, Lisboa, Portugal.

\section{Correspondência:}

Universidade Lusófona - Faculdade de Educação Física e Desporto. Campo Grande, 376, sala H.1.2. 1749-024 Lisboa, Portugal. luis.massuca@gmail.com

\section{RESUMO}

Objetivo: Estudar o comportamento do sexo e os efeitos da idade e da massa gorda sobre a capacidade aeróbia de jovens adolescentes. Métodos: Os 621 estudantes do ensino secundário participantes no estudo (14 aos 17 anos; feminino: $n=329$, idade, 15,84 \pm 0,92 anos; masculino: $n=292$, idade, 15,82 \pm 0,87 anos) foram avaliados em duas categorias: morfologia (altura, peso e \% massa gorda - \%MG) e aptidão física (capacidade aeróbia). As medições antropométricas foram realizadas de acordo com o protocolo descrito por Marfell-Jones e a \%MG foi calculada por

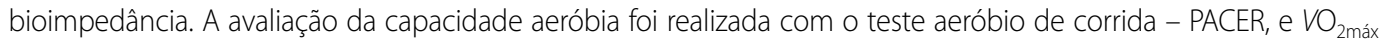
relativo foi calculado utilizando a equação de Léger. Os resultados das avaliações foram classificados de acordo com os valores normativos das tabelas de referência da bateria de testes FITNESSGRAM ${ }^{\circledR}$ As técnicas estatísticas utilizadas foram: 1) cálculo de frequências; 2) teste $t$ de Student para amostras independentes; e 3) ANOVA two-way seguida do teste post-hoc HSD de Bonferroni. Resultados: 1) existem diferenças significativas entre sexos no que se refere à \%MG e ao $\mathrm{VO}_{2 \text { máxi }}$ 2) durante a adolescência, o $\mathrm{VO}_{2 \text { máx }}$ estabiliza nos rapazes e sofre um declínio nas moças; 3 ) independentemente do sexo, a classe de \%MG e a idade cronológica têm um efeito significativo sobre a capacidade aeróbia; e 4) em jovens adolescentes, com \%MG de risco, a redução da \%MG para níveis saudáveis parece resultar na melhoria da capacidade aeróbia.Conclusão: O impacto da \%MG na capacidade aeróbia, reforça a importância da educação física escolar na promoção da saúde cardiovascular.

Palavras-chave: obesidade, aptidão física, saúde, adolescente.

\begin{abstract}
Objective: To study the behavior of sex and age and fat mass effects on aerobic capacity of young adolescents. Methods: The 621 students participating in the study (14 to 17 years, female: $n=329$, age $15.84 \pm 0.92$ years, male: $n=292$, age $15.82 \pm 0.87$ years) were evaluated in two categories: morphology (height, body mass and \% fat mass - \%FM) and physical fitness (aerobic capacity). The anthropometric measurements were performed according to the protocol described by Marfell-Jones and \%FM was calculated by bioelectrical impedance. The assessment of aerobic capacity was performed with the aerobic running test (PACER), and relative $\mathrm{VO}_{2 \max }$ was calculated using the equation of Leger. The evaluation results were classified according to the normative values of the FITNESSGRAM ${ }^{\circledR}$ reference tables. The statistical techniques used were: 1) calculation of frequencies; 2) Student t-test for independent samples; and 3) two-way ANOVA followed by post-hoc Bonferroni HSD. Results: 1) there are significant differences between genders with regard to $\% \mathrm{FM}$ and $\mathrm{VO}_{2 \text { maxi }}{ }^{2}$ ) during adolescence a stabilization of $\mathrm{VO}_{2 \max }$ was observed in boys and a decline in girls; 3) regardless of gender, the class of $\%$ FM and chronological age have a significant effect on aerobic capacity; and 4) in young people (adolescents) with \%FM risk, the reduction of \%FM to healthy levels seems to result in improved aerobic capacity. Conclusion: The impact of \%FM in aerobic capacity reinforces the importance of physical education in promoting cardiovascular health.
\end{abstract}

Keywords: obesity, physical fitness, health, adolescent.

\section{RESUMEN}

Objetivo: Estudiar el comportamiento del sexo, y los efectos de la edad y la mas a de tejido adiposo sobre la capacidad aeróbica de jóvenes adolescentes. Métodos: Los 621 estudiantes de enseñanza secundaria que participaron en el estudio (14 a 17 años; mujeres: $n=329$, promedio de edad, 15,84 \pm 0,92 años; hombres: $n=292$, promedio de edad, 15,82 \pm 0,87 años) fueron evaluados en dos categorías: morfología (altura, peso y \% de masa de tejido adiposo - \% MTA) y aptitud física (capacidad aeróbica). Las mediciones antropométricas fueron realizadas de acuerdo con el protocolo descrito por Marfell-Jones y el \% MTA fue calculado por bioimpedancia. La evaluación de la capacidad aeróbica se realizó mediante la prueba aeróbica de carrera - PACER, y el VO $\mathrm{Z}_{\text {max }}$ relativo se calculó utilizando la ecuación de Léger. Los resultados de las evaluaciones fueron clasificados de acuerdo con los valores normativos de las tablas de referencia de la batería de pruebas FITNESSGRAM ${ }^{\circledR}$. Las técnicas estadísticas utilizadas fueron: 1) cálculo de frecuencias; 2) prueba t de Student para muestras independientes; y 3) ANOVA two-way seguida de la prueba post-hoc HSD de Bonferroni. Resultados: 1) hay diferencias significativas entre sexos en lo que se refiere al \% MTA y al $\mathrm{VO}_{2 \text { maxi }}$ 2) durante la adolescencia, el $\mathrm{VO}_{2 \max }$ se estabiliza en los muchachos y sufre una reducción en las chicas; 3) independientemente del sexo, el tipo del \% MTA y la edad cronológica tienen un efecto significativo sobre la capacidad aeróbica; y 4) en jóvenes adolescentes, con \% MTA de riesgo, la disminución del \% MTA 
para niveles saludables parece resultar en la mejoría de la capacidad aeróbica. Conclusión: El impacto del \% de MTA, en la capacidad aeróbica, refuerza la importancia de la educación física escolar en la promoción de la salud cardiovascular.

Palabras clave: obesidad, aptitud física, salud, adolescente.

Artigo recebido em 03/02/2012, aprovado em 21/10/2013.

\section{INTRODUÇÃO}

A atividade física, a capacidade aeróbia e a composição corporal têm sido identificadas como importantes preditores de saúde em crianças e adolescentes ${ }^{1}$. Esta relação causal entre a atividade física (exercício), a aptidão física (capacidades motoras) e a qualidade de vida justificou o investimento de inúmeros países ou organizações no conhecimento/ rastreio da aptidão física das suas populações.

Dentre as capacidades motoras que caracterizam a aptidão física, a capacidade aeróbia é reconhecida como uma das mais importantes na promoção de saúde², sendo mesmo apontada como preditora (na infância) do nível de atividade física na idade adulta ${ }^{3}$

A diversidade de termos utilizados para a designar esta capacidade motora dificulta, por vezes, a sua compreensão. No entanto, parece que os termos resistência cardiorespiratória, capacidade de trabalho aeróbico e capacidade de trabalho físico surgem geralmente associados à capacidade de desempenho (i.e., capacidade para realizar grande atividade muscular por um período prolongado de tempo), enquanto o termo capacidade aeróbica $\left(\mathrm{VO}_{2 \text { máx }}\right)$ se refere a uma capacidade funcional propriamente dita (i.e., fisiológica).

De fato, o estudo do efeito do exercício na elevação da saúde ${ }^{4}$ permitiu identificar o $\mathrm{VO}_{2 \text { máx }}$ como o melhor indicador da "fitness" cardiorrespiratório 5 . Em outras palavras, $\mathrm{VVO}_{2 \text { máx }}$ reflete a taxa máxima de oxigênio que pode ser captado, fixado, transportado e utilizado pelo organismo (por unidade de tempo) durante um esforço máximo de características gerais ${ }^{6}$, i.e., reflete a capacidade global dos sistemas cardiovascular, respiratório e muscular esquelético.

Em complemento, o recurso a técnicas estatísticas de regressão e/ou alométricas pode ser identificado na literatura em estudos centrados no efeito da massa livre de gordura (MLG) ${ }^{7}$, índice de massa corporal $(\mathrm{IMC})^{8}$ e sexo $\left(\mathrm{ml} / \mathrm{kg}^{-0.67} \cdot \mathrm{min}^{-1}\right)^{9}$ sobre o $\mathrm{VO}_{2 \text { máx }}$. De fato, a literatura sugere que a MLG está fortemente correlacionada $(r=0,87) \mathrm{com}_{\mathrm{V}} \mathrm{VO}_{2 \text { máx }}$ de jovens com idades pré- pubertárias ${ }^{7}$, sendo mesmo sugerida a normalização do $\mathrm{VO}_{2 \text { máx }}$ pela $\mathrm{MLG}^{10}$ (uma vez que esta é metabolicamente mais ativa que a massa gorda).

Contudo, estudos recentes sugerem que a massa gorda não tem qualquer efeito no $\mathrm{VO}_{2 \text { máx }}$ de jovens com idades pré-pubertárias ${ }^{7}$ e pubertárias ${ }^{11}$. No entanto, a relação entre a capacidade aeróbia (em crianças e jovens) com os fatores de risco das doenças cardiovasculares ${ }^{12}$, em complemento com a conhecida associação entre a obesidade e as doenças cardiovasculares ${ }^{13}$ suscitam dúvidas.

Face ao exposto, é objetivo deste estudo investigar esse efeito, i.e., estudar o comportamento do sexo e os efeitos da idade cronológica e da massa gorda sobre a capacidade aeróbia en jovens adolescentes.

\section{MÉTODOS}

\section{Amostra}

Participaram no estudo 621 estudantes do ensino secundário (idade, 14 a 17 anos), sendo 329 do sexo feminino (idade, 15,8 0,9 anos) e 292 do sexo masculino (idade, 15,8 \pm 0,9 anos). O protocolo experimental segue a resolução do Conselho Nacional de Saúde (n 196/96) e foi aprovado pelo Conselho de Ética e Conselho Científico locais. O protocolo experimental foi apresentado a todos encarregados de educação que, depois de esclarecidos e garantida a proteção de privacidade dos voluntários, assinaram o termo de consentimento informado. Todos os participantes foram avaliados durante o mês de setembro de 2009 no pavilhão gimnodesportivo da mesma instituição de ensino e em condições de realização semelhantes.

\section{Avaliação morfofuncional}

A avaliação realizada abrangeu duas categorias, i.e., morfologia (proporcionalidade e composição corporal) e aptidão física (capacidade aeróbia).

Avaliação morfológica - Foram realizadas duas medições antropométricas, nomeadamente altura total ou estatura $(\mathrm{cm})$ e massa corporal ou peso $(\mathrm{kg})$, obtidas por antropometristas credenciados pela International Society of the Advance of Kinanthropometry (ISAK). No entanto, antes das medições dos sujeitos, foi calculada a garantia absoluta ou erro padrão de medida (TEM) dos antropometristas. O protocolo seguido foi o descrito por Marfell-Jones et al..$^{14}$ e foram utilizados instrumentos portáteis. Assim, para a medição da estatura e altura sentada utilizou-se um antropômetro (Anthropometric Kit Siber-Hegner Machines SA GPM, 2008) e para a massa corporal utilizou-se uma balança (Body Mass Scale Vogel \& Halke, Secca model 761 7019009, Alemanha, 2006) que permite leituras de 500 em $500 \mathrm{~g}$. No estudo da composição corporal foi considerada a percentagem de massa gorda predita por bioimpedância. A bioimpedância foi realizada utilizando aparelho Quantum BIA-101Q ${ }^{\circledR}$ (RJL Systems, Inc. Clinton: MI, EUA), com uma frequência de $50 \mathrm{kHz}$ em corrente alternada de quatro eletrodos. A classificação dos sujeitos teve como referências os valores normativos adoptados pelo Instituto Cooper ${ }^{15}$ apresentados na tabela 1.

Tabela 1. Normas para a zona saudável de massa gorda (adaptado de The Cooper Institute ${ }^{15}$ ).

\begin{tabular}{c|c|c|c|c|c}
\hline \multirow{3}{*}{ Sexo } & \multirow{2}{*}{ Idade } & \multicolumn{4}{|c}{ \% Massa gorda } \\
\cline { 4 - 6 } & \multirow{2}{*}{ Muito leve } & \multirow{2}{*}{ Saudável } & \multicolumn{2}{|c}{ Precisa melhorar } \\
\cline { 4 - 6 } & & & & Risco moderado & Risco elevado \\
\hline \multirow{4}{*}{ Masculino } & 14 & $\leq 7,0$ & $7,1-21,3$ & $21,4-33,1$ & $\geq 33,2$ \\
\cline { 2 - 6 } & 15 & $\leq 6,5$ & $6,6-20,1$ & $20,2-31,4$ & $\geq 31,5$ \\
\cline { 2 - 6 } & 16 & $\leq 6,4$ & $6,5-20,1$ & $20,2-31,5$ & $\geq 31,6$ \\
\cline { 2 - 6 } & 17 & $\leq 6,6$ & $6,7-20,9$ & $21,0-32,9$ & $\geq 33,0$ \\
\hline \multirow{5}{*}{ Feminino } & 14 & $\leq 13,9$ & $14,0-28,5$ & $28,6-36,7$ & $\geq 36,8$ \\
\cline { 2 - 6 } & 15 & $\leq 14,5$ & $14,6-29,1$ & $29,2-37,0$ & $\geq 37,1$ \\
\cline { 2 - 6 } & 16 & $\leq 15,2$ & $15,3-29,7$ & $29,8-37,3$ & $\geq 37,4$ \\
\cline { 2 - 6 } & 17 & $\leq 15,8$ & $15,9-30,4$ & $30,5-37,8$ & $\geq 37,9$ \\
\hline
\end{tabular}

Avaliação da capacidade aeróbia - $O$ teste aeróbio de corrida vai-e-vem de 20 m (ou PACER), permite estudar a resposta ao exercício submáximo, com recurso a um protocolo progressivo submáximo (por níveis) sem interrupção do esforço. Este teste tem um alto teor de validade $(0,51-0,90)$ e reprodutibilidade (0,75-0,93). Para a realização do teste foi necessário: um local plano de pelo menos 25 metros, um leitor de CDs, um CD com as cadências, quatro cones e folhas de anotação. $\mathrm{O}$ teste pode ser aplicado a grupos de seis a 10 participantes em simultâneo que, correndo juntas a um ritmo cadenciado pelo sinal sonoro, devem correr um percurso de 20 $\mathrm{m}$, delimitado entre duas linhas paralelas. A banda sonora emite bipes, a intervalos específicos para cada nivel, sendo que a cada bipe o avaliado deverá ter ultrapassado com um dos pés numa das linhas paralelas e voltar em sentido contrário. No primeiro nível a velocidade é de $8,5 \mathrm{~km} / \mathrm{h}$, sendo acrescida de $0,5 \mathrm{~km} / \mathrm{h}$ a cada um dos níveis seguintes. Esta mudança do nível é sinalizada por uma voz, e o teste termina quando o avaliado não consegue manter a cadência necessária à realização do percurso. 0 valor do $\mathrm{VO}_{2 \text { máx }}\left(\mathrm{ml} \cdot \mathrm{kg}^{-1} \cdot \mathrm{min}^{-1}\right)$ foi calculado utilizando a equação: 31,025 + 3,238(Vel)-3,248(ld)+0,1536(ld $\cdot V e l),\left(R=0,71, S E M=5,9 \mathrm{ml} \cdot \mathrm{kg}^{-1} \cdot \mathrm{min}^{-1}\right)$, sendo: Vel, velocidade em $\mathrm{km} / \mathrm{h}$ no nível atingido; Id, idade do sujeito em anos ${ }^{16}$. Posteriormente, e à semelhança do realizado anteriormente, os resultados foram transformados em valores normativos de acordo com as tabelas de referência da bateria de testes FITNESSGRAM ${ }^{\circ 15}$ (tabela 2). 
Tabela 2. Normas para a zona saudável de capacidade aeróbia (adaptado de The Cooper Institute ${ }^{15}$.

\begin{tabular}{c|c|c|c|c}
\hline \multirow{3}{*}{ Sexo } & \multirow{2}{*}{ Idade } & \multicolumn{3}{|c}{ VO $_{\text {2máx }}\left(\mathbf{m l} \cdot \mathbf{k g}^{-1} \cdot \mathbf{m i n}^{-1}\right)$} \\
\cline { 3 - 5 } & & \multirow{2}{*}{ Saudável } & \multicolumn{2}{|c}{ Precisa melhorar } \\
\cline { 3 - 5 } & & & Risco moderado & Risco elevado \\
\hline \multirow{4}{*}{ Masculino } & 14 & $\geq 42,5$ & $42,4-39,7$ & $\leq 39,6$ \\
\cline { 2 - 5 } & 15 & $\geq 43,6$ & $43,5-40,7$ & $\leq 40,6$ \\
\cline { 2 - 5 } & 16 & $\geq 44,1$ & $44,0-41,1$ & $\leq 41,0$ \\
\cline { 2 - 5 } & 17 & $\geq 44,2$ & $44,1-41,3$ & $\leq 41,2$ \\
\hline \multirow{4}{*}{ Feminino } & 14 & $\geq 39,4$ & $39,3-36,4$ & $\leq 36,3$ \\
\cline { 2 - 5 } & 15 & $\geq 39,1$ & $39,0-36,1$ & $\leq 36,0$ \\
\cline { 2 - 5 } & 16 & $\geq 38,9$ & $38,8-35,9$ & $\leq 35,7$ \\
\cline { 2 - 5 } & 17 & $\geq 38,8$ & $38,7-35,8$ & \\
\hline
\end{tabular}

\section{Análise estatística}

Após a organização das observações das variáveis contínuas em estudo (\%MG e VO $\mathrm{Vmáx}_{2}$, contempladas nas tabelas de referência da bateria de testes FITNESSGRAM ${ }^{\circ}{ }^{15}$, foram calculadas as frequências de cada uma das classes na amostra e, com recurso a histogramas de frequências, representadas graficamente. A significância da diferença entre a \%MG, $\mathrm{VO}_{2 \text { máx }}$ com os participantes do sexo feminino vs. sexo masculino foi avaliada com o teste $t$ de Student para amostras independentes. Os pressupostos de normalidades das distribuições e a homogeneidade de variâncias nos dois grupos foram avaliados com: teste de Kolmogorov-Smirnov com correção de Lilliefors (\%MG: KS(329) feminino $=0,054$; $p=0,023 ; \mathrm{KS}(292)_{\text {masculino }}=0,098 ; p<0,001 ; \mathrm{VO}_{2 \text { máx: }}: \mathrm{KS}(329)_{\text {feminino }}=0,108 ; p<$ 0,$\left.001 ; \mathrm{KS}(292)_{\text {masculino }}=0,078 ; p<0,001\right)$; e teste de Levene (\%MG: $F(1,619)=$ $\left.0,956, p=0,329 ; V_{2 \text { máx: }} F(1.554,868)=13,715, p<0,001\right)$. Os resultados são apresentados como média (M), desvio padrão (DP), erro padrão da média (SEM), e apresenta-se como medida de dimensão de efeito o d de Cohen. Para avaliar se a idade cronológica (Idade) e se a classe de percentagem de massa gorda (\%MG (Classe)) afetam significativamente a capacidade aeróbia $\left(\mathrm{VO}_{2 \text { máx }}\right)$ recorreu-se à ANOVA two-way seguida do teste post-hoc HSD de Bonferroni. O pressuposto da distribuição normal da variável dependente $\left(\mathrm{VO}_{2 \text { máx }}\right)$ nos diferentes grupos definidos pelo cruzamento dos fatores "Idade" se)" também foi avaliado pelo teste de KS com correção de Lilliefors. Em 50\% dos grupos obteve-se $p<0,1$, mas na generalidade o desvio à normalidade é pequeno. Uma vez que a ANOVA é robusta a violações suaves deste pressuposto, não foi necessário proceder a transformações matemáticas corretivas. O pressuposto de homogeneidade de variância foi validado com o teste de Levene quando estudado o $\mathrm{VO}_{2 \text { máx }}$ (feminino: $F(15,313)=1,959, p=0,018$; masculino: $F(14,277)=1,606, p=0,077)$. As análises estatísticas descritivas, gráficas e inferenciais foram executadas com o programa informático Statistical Package for The Social Sciences (SPSS Inc, v.17.0, Chicago, Illinois). Considerou-se estatisticamente significativo o efeito cujo $p$-value foi inferior ou igual a 0,05.

\section{RESULTADOS}

As observações das variáveis contínuas em estudo são organizadas em classes no eixo das abcissas e a frequência de cada uma dessas classes na amostra é representada no eixo das ordenadas (figura 1). A figura 1A permite constatar que 31,2\% dos participantes se situam fora da classe correspondente a uma \%MG considerada saudável (masculino, 13,6\%; feminino, 17,6\%). Quando considerada a capacidade aeróbia (figura 1B), observa-se que pouco mais de metade da amostra se situa na classe correspondente à zona saudável $(54,3 \%)$. Em particular, 34,0\% $(n=112)$ das moças surgem no grupo de alto risco e $27,1 \%(n=89)$ no grupo de risco moderado $(61,1 \%$ revelam uma capacidade aeróbia de risco para a saúde). Também 28,4\% dos rapazes revelam uma capacidade aeróbia de risco para a saúde $(11,0 \%(n=32)$ de risco moderado e $17,4 \%(n=51)$ de alto risco).

O estudo da significância permite constatar que os rapazes têm uma \%MG de, em média, 13,39\% (SEM =0,39), enquanto as moças revelam, em média, $25,12 \%$ (SEM =0,39). De acordo com o teste $t$ de Student, as diferenças de \%MG observadas entre os dois grupos são estatisticamente significativas $(t(619)=$ 21,$208 ; p<0,001$ ) (tabela 3). A dimensão do efeito do sexo é muito elevada $(d=-1,71)$ e, de acordo com o I.C. a 95\% ]10,64;12,82[, as moças têm, em

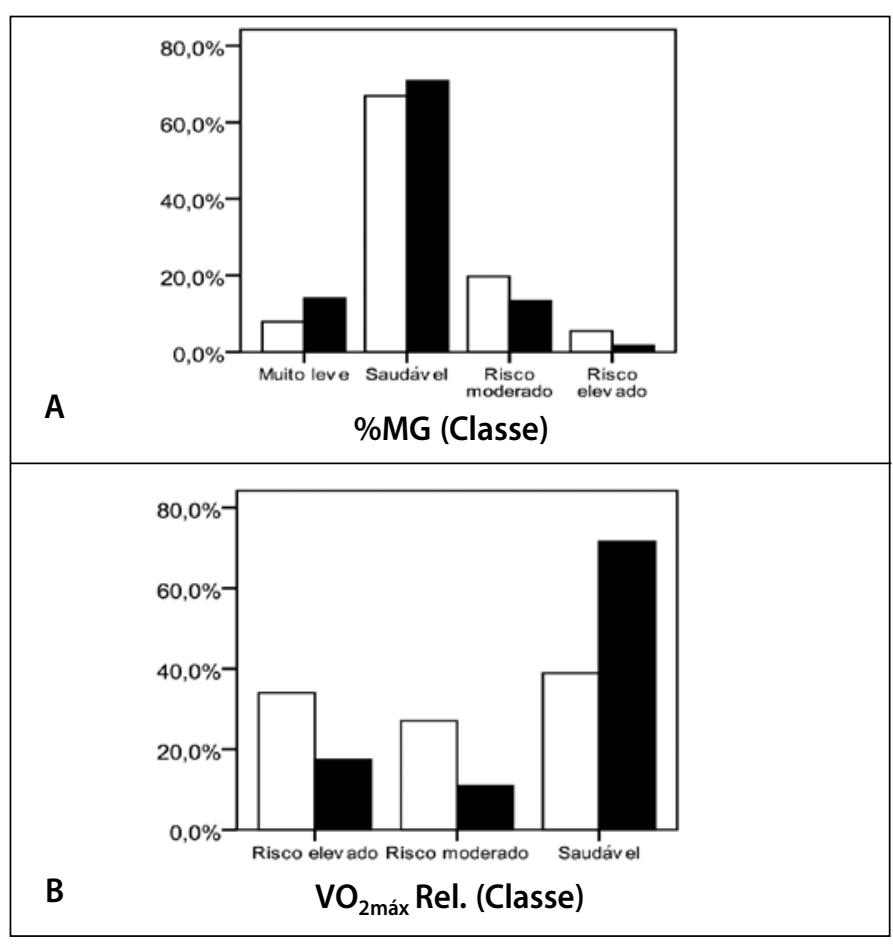

Figura 1. Histogramas, de frequências por sexo (masculino, $n=292$; feminino, $n=329$ ), para as escalas de medida ordinal percentagem de massa gorda (\%MG; $\mathrm{A}) \mathrm{VVO}_{2 \text { máx }}$ relativo (B).

Tabela 3. Valores médios e desvio padrão das variáveis percentagem de massa gorda e do $\mathrm{VO}_{2 \text { máx }}$ relativo nos dois grupos (sexo masculino vs. sexo feminino) dos 14 aos 17 anos de idade.

\begin{tabular}{|c|c|c|c|c|c|c|c|c|c|c|}
\hline \multirow{2}{*}{ Idade } & \multirow{2}{*}{ Sexo } & \multirow[b]{2}{*}{10} & \multicolumn{4}{|c|}{$\%$ MG } & \multicolumn{4}{|c|}{$\mathrm{VO}_{2 \text { máx }}\left(\mathrm{ml} \cdot \mathrm{kg}^{-1} \cdot \mathrm{min}^{-1}\right)$} \\
\hline & & & M & DP & SEM & $t(p)$ & M & DP & SEM & $t(p)$ \\
\hline \multirow{2}{*}{14} & Fem. & 27 & 27,98 & 7,01 & 1,35 & \multirow{2}{*}{$7,777(* * *)$} & 41,33 & 4,28 &, 82 & \multirow{2}{*}{$-3,733(* *)$} \\
\hline & Masc. & 17 & 12,45 & 5,42 & 1,32 & & 48,31 & 6,92 & 1,68 & \\
\hline \multirow{2}{*}{15} & Fem. & 87 & 25,79 & 6,60 &, 71 & \multirow{2}{*}{$12,586(* * *)$} & 38,20 & 3,81 & .41 & \multirow{2}{*}{$-12,504\left(^{*}\right.$} \\
\hline & Masc. & 91 & 13,46 & 6,47 & ,68 & & 47,35 & 5,79 &, 61 & \\
\hline \multirow{2}{*}{16} & Fem. & 125 & 25,69 & 6,45 &, 58 & \multirow{2}{*}{$12,808(* * *)$} & 37,47 & 4,05 &, 36 & \multirow{2}{*}{$-14,877$} \\
\hline & Masc. & 113 & 14,49 & 7,03 & ,66 & & 46,04 & 4,83 & .45 & \\
\hline \multirow{2}{*}{17} & Fem. & 90 & 22,82 & 7,88 & 83 & \multirow{2}{*}{$9,689(* * *)$} & 36,41 & 4,88 & .51 & \multirow{2}{*}{$-11,849{ }^{*}$} \\
\hline & Masc. & 71 & 11,77 & 6,19 & ,74 & & 46,49 & 5,91 &, 70 & \\
\hline \multirow{2}{*}{ Total } & Fem. & 329 & 25,12 & 7,09 & 39 & \multirow{2}{*}{21,208 ( $^{*}$} & 37,69 & 4,42 & 24 & \multirow{2}{*}{$-22,149$ (** $^{* *}$} \\
\hline & Masc. & 292 & 13,39 & 6,63 & , 39 & & 46,69 & 5,55 &, 32 & \\
\hline
\end{tabular}

*** $p<0,001 ; * * 0<0,01$

média, uma \%MG superior (entre 10,64 e 12,82\%) à dos rapazes. Quando considerados os grupos etários, observou-se que a diferença entre os sexos também é estatisticamente significativa (14 anos: $t(42)=7,777, p<0,001$, I.C. a 95\%, ]11,50;19,56[; 15 anos: $t(176)=12,586, p<0,001$, I.C. a 95\%, ]10,39;14,26[; 16 anos: $t(236)=12,808, p<0,001$, I.C. a 95\%, ]9,48;12,92[; 17 anos: $t(159)$ $=9,689, p<0,001$, I.C. a 95\%, ]8,80;13,31[), e que as moças com 14, 15, 16 e 17 anos têm, em média, uma \%MG superior aos rapazes (entre 8,80\% aos 17 anos e 19,56\% aos 14 anos). Por sua vez, os rapazes têm um $V_{2 m a ́ x}$ estatisticamente superior $(t(554,868)=-22,149 ; p<0,001)$ ao das moças $\left(\mathrm{M}=46,69 \mathrm{ml} \cdot \mathrm{kg}^{-1} \cdot \mathrm{min}^{-1}, \mathrm{SEM}=0,32 ; \mathrm{M}=37,69 \mathrm{ml} \cdot \mathrm{kg}^{-1} \cdot \mathrm{min}^{-1}, \mathrm{SEM}=0,24\right.$, respectivamente), i.e., entre 8,20 e $9,80 \mathrm{ml}^{\mathrm{kg}} \mathrm{kg}^{-1} \cdot \mathrm{min}^{-1}$. De fato, a dimensão do efeito do sexo é muito elevada $(d=1,8)$. Quando considerados os grupos etários, observou-se que a diferença entre os sexos também é estatisticamente significativa (14 anos: $t(23,790)=-3,733, p=0,001$, I.C. a 95\%, ]-10,84; -3,12[; 15 anos: $t(156,393)=-12,504, p<0,001$, I.C. a 95\%, ]-10,59;-7,70[; 16 anos: $t(236)=-14,877, p<0,001$, I.C. a 95\%, ]-9,71;-7,44[; 17 anos: $t(156,393)=$ $-12,504, p<0,001$, I.C. a 95\%, ]-11,76;-8,40[), e que as moças com 14, 15, 16 e 17 anos têm, em média, uma capacidade aeróbia inferior aos rapazes.

Estudando isoladamente o grupo de participantes do sexo feminino, observou-se que, depois de considerados os efeitos da classe de \%MG, é possivel afirmar que a idade cronológica teve um efeito estatisticamente 
significativo, de médio efeito e de elevada potência sobre a capacidade aeróbia $\left(F(3,322)=13,490 ; p<0,001 ; \eta_{p}^{2}=0,112\right.$; potência $\left.=1,000\right)$. $O$ grupo de moças com 17 anos apresenta o menor valor médio de $\mathrm{VO}_{2 \text { máx }}(M=35,18$ $\mathrm{ml} \cdot \mathrm{kg}^{-1} \cdot \mathrm{min}^{-1} ;$ SEM $\left.=0,50 ; n=90\right)$, seguido pelos grupos de moças com 16 anos $\left(M=36,38 \mathrm{ml} \cdot \mathrm{kg}^{-1} \cdot \mathrm{min}^{-1} ;\right.$ SEM $\left.=0,47 ; n=125\right), 15$ anos $\left(M=37,28 \mathrm{ml} \cdot \mathrm{kg}^{-1} \cdot \mathrm{min}^{-1}\right.$;

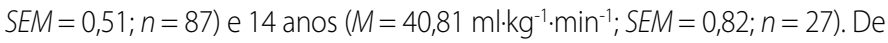
acordo com o teste post-hoc de Bonferroni, as diferenças significativas para este fator ocorrem entre o grupo de moças de 14 anos e todos os grupos etários estudados (15 anos: I.C. a 95\% ]1,125;5,935[, $p=0,001 ; 16$ anos: I.C. a 95\% ]2,103;6,755[, $p<0,001 ; 17$ anos: I.C. a 95\% ]3,204;8,059[, $p<0,001)$, e entre os grupos de moças de 15 e 17 anos (I.C. a 95\% ]0,445;3,757[; $p=0,005$ ).

Relativamente ao grupo do sexo masculino, depois de considerados os efeitos da classe de \%MG, observou-se que a idade cronológica não teve um efeito estatisticamente significativo sobre a capacidade aeróbia $(F(3,285)$ $=43,921 ; p=0,152 ; \eta_{p}^{2}=0,018 ;$ potência =0,461). À semelhança do que se observou para as moças, também nos rapazes, o grupo com 17 anos apresenta o menor valor de $\mathrm{VO}_{2 \text { máx }}\left(M=43,01 \mathrm{ml} \cdot \mathrm{kg}^{-1} \cdot \mathrm{min}^{-1} ; \mathrm{SEM}=0,82\right.$; $n=71)$, seguido pelos grupos com 16 anos $\left(M=43,75 \mathrm{ml} \cdot \mathrm{kg}^{-1} \cdot \mathrm{min}^{-1}\right.$; SEM $=0,72 ; n=113), 15$ anos $\left(M=44,68 \mathrm{ml} \cdot \mathrm{kg}^{-1} \cdot \mathrm{min}^{-1} ;\right.$ SEM $\left.=0,76 ; n=91\right)$ e 14 anos $\left(M=44.96 \mathrm{ml}^{\prime} \mathrm{kg}^{-1} \cdot \mathrm{min}^{-1} ;\right.$ SEM $\left.=1,36 ; n=17\right)$. Contudo, e de acordo com o teste post-hoc de Bonferroni, as diferenças observadas entre os grupos $(14,15,16$ e 17 anos) não são estatisticamente significativas. Os resultados são apresentados na figura 2.

De modo semelhante, depois de considerar o efeito da idade cronológica, observou-se que a classe de \%MG influenciou significativamente a capacidade aeróbia das moças, sendo o efeito de média dimensão e a potência do teste elevada $\left(F(3,322)=9,493 ; p<0,001 ; \eta_{p}^{2}=0,081\right.$; potência $=0,997)$. As moças pertencentes à classe de "risco elevado" apresentam, em média, os menores valores de $\mathrm{VO}_{2 \text { máx }}\left(M=35,34 \mathrm{ml} \cdot \mathrm{kg}^{-1} \cdot \mathrm{min}^{-1} ; \mathrm{SEM}=\right.$ $0,97 ; n=18)$, a que se seguem as das classes "risco moderado" $(M=36,76$ $\mathrm{ml} \cdot \mathrm{kg}^{-1} \cdot \mathrm{min}^{-1} ;$ SEM $\left.=0,52 ; n=65\right)$, "muito leves" $\left(M=38,35 \mathrm{ml} \cdot \mathrm{kg}^{-1} \cdot \mathrm{min}^{-1}\right.$; SEM $=0,83 ; n=26)$ e "saudável" ( $M=38,22 \mathrm{ml} \cdot \mathrm{kg}^{-1} \cdot \mathrm{min}^{-1} ;$ SEM =0,32; $n=220$ ). De acordo com o teste post-hoc de Bonferroni, as diferenças estatisticamente significativas para este fator ocorrem entre as moças da classe "saudável" e as duas classes de risco ("risco moderado": I.C. a 95\% ]0,905;4,011[, $p<0,001$; "risco elevado": I.C. a 95\% ]1,201;6,561[, $p=0,001)$.

Observou-se ainda que, depois de considerado o efeito da idade cronológica, a classe de \%MG também influenciou significativamente a capa-

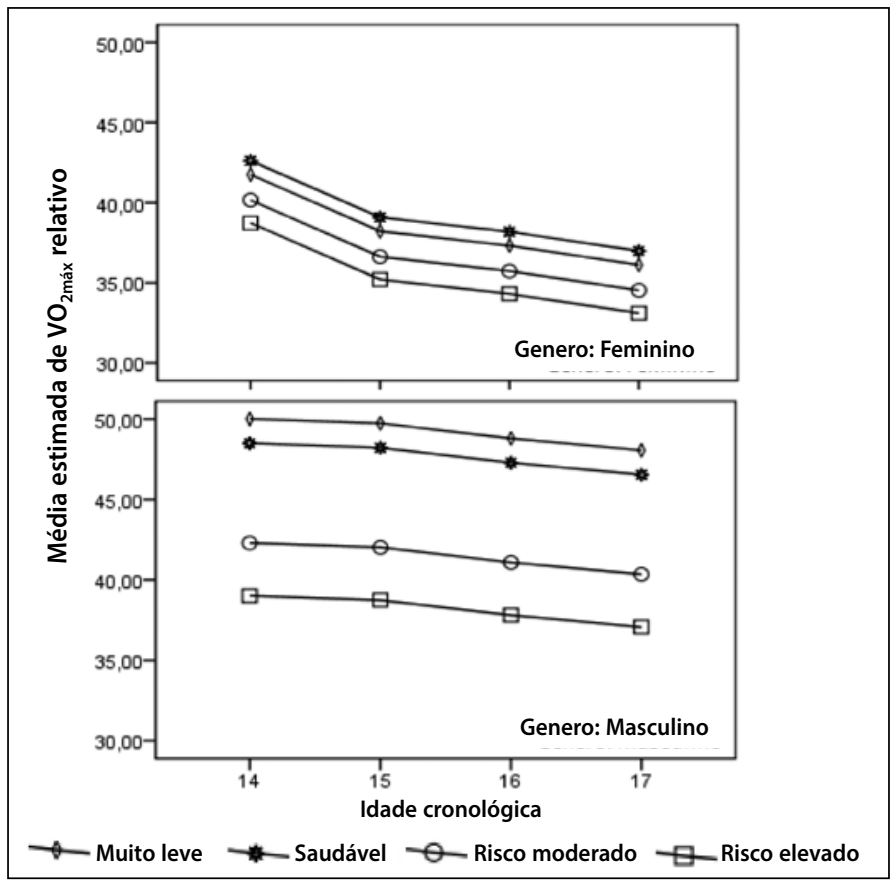

Figura 2. Capacidade aeróbia (estimada), por sexo, nos grupos etários dos 14 aos 17 anos de idade. cidade aeróbia dos rapazes, sendo a dimensão do efeito e a potência do teste elevadas $\left(F(3,285)=598,879 ; p<0,001 ; \eta_{p}^{2}=0,203\right.$; potência $\left.=1,000\right)$. Assim, os rapazes pertencentes à classe de "risco elevado" apresentam, em média, o menor valor de $\mathrm{VO}_{2 \text { máx }}\left(M=38,16 \mathrm{ml} \cdot \mathrm{kg}^{-1} \cdot \mathrm{min}^{-1} ; \mathrm{SEM}=2,25 ; n=5\right)$, seguido pelos rapazes que integram as classes "risco moderado" $(M=41,44$ $\mathrm{ml} \cdot \mathrm{kg}^{-1} \cdot \mathrm{min}^{-1} ;$ SEM $\left.=0,86 ; n=39\right)$, "saudável" $\left(M=47,63 \mathrm{ml} \cdot \mathrm{kg}^{-1} \cdot \mathrm{min}^{-1} ;\right.$ SEM = $0,42 ; n=207)$ e "muito leves" ( $\left.M=49,16 \mathrm{ml} \cdot \mathrm{kg}^{-1} \cdot \mathrm{min}^{-1} ; \mathrm{SEM}=0,82 ; n=41\right)$. De acordo com o teste post-hoc de Bonferroni, as diferenças estatisticamente significativas para este fator ocorrem entre os rapazes das classes "muito leve" e "saudável" com as duas classes de risco ("muito leve" vs. "risco moderado": I.C. a 95\% ]4,697;10,730[, $p<0,001$; "muito leve" vs. "risco elevado": I.C. a 95\% ]4,724;17,267[ $p<0,001$; "saudável" vs. "risco moderado": I.C. a 95\% ]3,852;8,541[ $p<0,001$; "saudável" vs. "risco elevado": I.C. a 95\% ]3,496;15,462[, $p<0,001$ ). Os resultados são apresentados graficamente na figura 3.

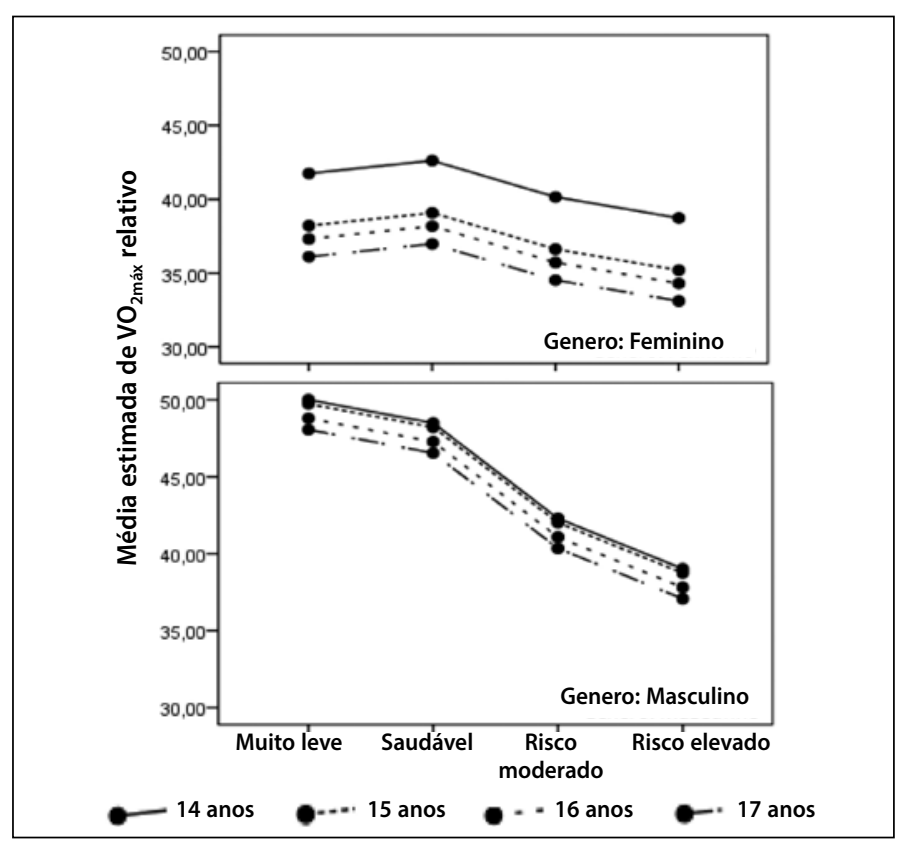

Figura 3. Capacidade aeróbia (estimada), por sexo, nas classes de percentagem de massa gorda.

\section{DISCUSSÃO}

A pertinência do estudo é reforçada quando se observa que $61,1 \%$ $(n=201)$ das moças e $28,4 \%(n=83)$ dos rapazes revelam um $V_{2 \text { máx }}$ de risco para a saúde (figura 1B). A literatura também já fazia prever a existência de diferenças de capacidade aeróbia entre sexos, sendo portanto expectável que as moças tivessem em média um $\mathrm{VO}_{2 \text { máx }}$ significativamente inferior (-8,20 a -9,80 $\mathrm{ml} \cdot \mathrm{kg}^{-1} \cdot \mathrm{min}^{-1}$ ) ao dos rapazes, mesmo quando considerados individualmente os quatro grupos etários em estudo (14, 15, 16 e 17 anos). Uma razão explicativa dos resultados prende-se com o fato de as moças adolescentes terem propensão a desenvolver uma deficiência em ferro ${ }^{17}$, com efeito no transporte de oxigênio, e com tradução em valores de $\mathrm{VO}_{2 \text { máx }}$ inferiores aos dos rapazes.

Observou-se, ainda, que a idade cronológica tem um efeito significativo sobre o $\mathrm{VO}_{2 \text { máx }}$ das moças (figura 2), uma vez que este decresce dos 14 para os 17 anos, o que enfatiza a importância do efeito da idade cronológica sobre a capacidade aeróbia. Segundo Astrand et al. ${ }^{6}$, nas moças verifica-se um contínuo decréscimo no $\mathrm{VO}_{2 \text { máx }}$ relativo ao longo de toda a vida (sendo superior na fase da adolescência), fato já referido em vários estudos longitudinais ${ }^{18}$, e que pode estar relacionado com: 1) o fato de o sistema de transporte de oxigênio não crescer tão rapidamente como a massa corporal; ou 2) o aumento da massa gorda durante a maturação sexual das moças ${ }^{19}$.

Embora os resultados deste estudo sejam concordantes com a literatura no que concerne às moças, já no que diz respeito aos rapazes, esperava-se que o $\mathrm{VO}_{2 \text { máx }}$ aumentasse com a idade ${ }^{15}$, principalmente durante a adolescência ${ }^{18}$. No entanto, constatou-se que embora $\mathrm{VO}_{2 \text { máx }}$ decresça ligeiramente 
com a idade cronológica, o efeito da idade não é significativo. De fato, as diferenças observadas sugerem que os rapazes aos 14 anos já atingiram o estado estável, sendo esta observação concordante com outros estudos realizados com rapazes entre 14 e 16 anos, que relatam a inexistência de alterações ${ }^{20}$, ou uma ligeira diminuição ${ }^{21}$ do $\mathrm{VO}_{2 m a ́ x}$.

Contudo, segundo Armstrong e Welsman²2, as análises tradicionais que utilizam a massa corporal total dos rapazes para relativizar o $\mathrm{VO}_{2 \text { máx }}$ têm reportado uma estabilização destes valores (entre 8 e 16 anos), enquanto estudos que utilizam técnicas alométricas para remover o efeito do tamanho corporal têm demonstrado que o $\mathrm{VO}_{2 \text { máx }}$ dos rapazes não se mantém estabilizado, mas sofre um ligeiro aumento. De fato, a estabilização do $\mathrm{VO}_{2 \text { máx }} \mathrm{Com}$ o decorrer da idade, sugere um crescimento proporcional entre a capacidade aeróbia relativa e o tamanho corporal nos rapazes ${ }^{23}$

Parece, portanto, existir uma relação entre o crescimento proporcional e as transformações (pubertárias) ocorridas ao nível dos sistemas respiratório e cardiovascular (pelo aumento do número de glóbulos vermelhos circulantes e consequente aumento da concentração de hemoglobina ${ }^{22}$ ), com repercussões favoráveis ao nível da captação, fixação e transporte de oxigênio, assim como do tamponamento sanguíneo ${ }^{24}$.

Face ao exposto, destacamos a pertinência da observação de Krahenbuhl e Williams ${ }^{23}$ ao referirem que (em crianças e jovens) o $\mathrm{VO}_{2 \text { máx }}$ (relativo) não serve como indicador útil da capacidade de resistência (pois não aumenta com a idade, enquanto a prestação de corrida melhora visivelmente). Ou seja, não traduz o crescimento de "reserva" aeróbia e a redução na utilização da $\% \mathrm{VO}_{2 \text { máx }}$ e do esforço relativo com o avançar da idade ${ }^{23}$.

Também se observou no início deste estudo que 31,2\% dos jovens se situaram fora da classe de \%MG considerada saudável (figura 1A), e que as moças têm, em média, uma \%MG significativamente superior (8,80\% aos 17 anos e 9,56\% aos 14 anos) aos rapazes (masculino, 13,39\%; feminino, 25,12\%). Assim, parece que a adiposidade constitui um fator determinante na diferenciação sexual (durante a adolescência). Este raciocínio resulta do conhecimento da influência das diferenças hormonais, evidente nos rapazes com o aumento de massa muscular e nas moças com o aumento de tecido adiposo ${ }^{25}$. De fato, o aumento na massa muscular dos rapazes possibilita uma maior utilização de oxigênio, e o aumento da \%MG que ocorre nas moças pode conduzir a uma diminuição da capacidade aeróbia.

Embora Goran et al. ${ }^{7}$ tenham sugerido que a massa gorda não tem qualquer efeito no $\mathrm{VO}_{2 \text { máx }}$ de jovens com idades pré-pubertárias, os resultados

\section{REFERÊNCIAS}

1. Rizzo NS, Ruiz JR, Hurtig-Wennlof A, Ortega FB, Sjostrom M. Relationship of physical activity, fitness, and fatness with clustered metabolic risk in children and adolescents: The European Youth Heart Study. J Pediatrics 2007;150:388-94

2. Andersen $\mathrm{LB}$, Hasseltrom $\mathrm{H}$, Gronfeldt $\mathrm{V}$, Hansen $\mathrm{S}$, Karsten $\mathrm{K}$. The relationship between physical fitness and clustered risk, and tracking of clustered risk from adolescence to young adulthood: Eight years follow-up in the Danish Youth and Sport Study. Int J Behav Nutr Phys Act 2004;1:6.

3. Kemper $\mathrm{H}$, Vente W, Mechelen W, Twisk J. Adolescent motor skills and performance: Is physical activity in adolescence related to adult physical fitness? Am J Hum Biol 2001;13:180-9.

4. Pffenbarger RS. Contributions of epidemiology to exercise science and cardiovascular health. Med Sci Sports Exerc 1988:20:426-38.

5. Buono MJ, Roby JJ, Micale FG, Sallis JF, Shepard WE. Validity and reliability of predicting maximum oxygen uptake via field tests in children and adolescents. Pediatr Exerc Sci 1991;3:250-5.

6. Astrand PO, Rodahl K, Dahl H, Stromme S. Textbook of workphysiology. New York: McGraw-Hill, 2003.

7. Goran M, Fields DA, Hunter GR, Herd SL, Weinsier RL. Total body fat does not influence maximal aerobic capacity. Int J Obes Relat Metab Disord 2000;24:841-8.

8. Ozcelik O, Aslan M, Ayar A, Kelestimur H. Effects of body mass index on maximal work production capacity and aerobic fitness during incremental exercise. Physiol Res 2004:53:165-70.

9. Janz KF, Mahoney LT. Three-years follow-up of charges in aerobic fitness during puberty: The Muscatine Study. Res Q for Exerc Sport 1997;68:1-9.

10. Janz KF, Burns TL, Witt JD, Mahoney LT. Longitudinal analysis of scaling $\mathrm{VO}_{2}$ for differences in body size during puberty: the Muscatine Study. Med Sci Sports Exerc 1998;30:1436-44.

11. Hands B, Larkin S, Parker H, Straker L, Perry M. The relationship among physical activity, motor competence and health-related fitness in 14-year-old adolescents. Scand J Med Sci 2009;19:655-63.

12. McMurray RG, Ainsworth $B E$, Harrell JS, Griggs TR, Williams OD. Is physical activity or aerobic power more influential on reducing cardiovascular disease risk factors? Med Sci Sports Exerc 1998:30:1520-9.

13. Williams DP, Going SB, Lohman TG, Harska DW, Srinivasan SR, Webber LS, et al. Body fatness and risk for elevated blood pressure,total cholesterol and serum lipoprotein ratios in children and adolescents. Am J Public Health 1992;82:358-63.

14. Marfell-Jones M, Olds T, Stewart A, Carter JEL. International Standards for Anthropometric Assessment (revised 2006). Underdale, S.A.: International Society for the Advanced of Kinanthropometry, 2006. ISBN 0-620-36207-3.

15. The Cooper Institute. Standards for Healthy Fitness Zone, Revision 8.6 and 9.x. Acessado em 10 setembro 2010 de: http://www.cooperinstitute.org/ourkidshealth/fitnessgram/documents/ Standardsv9final.pdf apresentados sugerem a existência de efeito significativo da classe de \%MG sobre o $\mathrm{VO}_{2 \text { máx }}$ de jovens de ambos os sexos. Esta observação é concordante com as de outros estudos mais recentes ${ }^{19,26-28}$ que reportam a existência de forte correlação entre a capacidade aeróbia e diversas medidas de avaliação da massa gorda em crianças e adolescentes, principalmente em sujeitos com \%MG correspondentes aos grupos de risco (moderado e elevado).

Evidentemente, a associação entre sujeitos com maior \%MG (risco moderado e elevado) com capacidades aeróbias inferiores, sugere uma diminuição da capacidade de suporte do próprio peso e consequentemente maior suceptibilidade de risco para a saúde. De fato, a \%MG parece estar relacionada com o desempenho no teste $\mathrm{PACER}^{29}$ e logicamente com um menor $\mathrm{VO}_{2 \text { máx }}$. predito $^{19}$.

É evidente que este estudo encerra limitações que se prendem com o fato de: o número de participantes, permitindo estatísticas próprias para grandes amostras, não ser representativo da população escolar adolescente; apesar de a prova de desempenho aeróbio ser de validade e fiabilidade reconhecida, não esgota a necessidade de estudos mais pormenorizados e intensivos, próprios de desenhos com amostras de menor dimensão e natureza laboratorial; e não ter sido considerado o efeito da maturação na variabilidade dos resultados apresentados.

\section{CONCLUSÃO}

A evidência de que jovens com \%MG de "risco moderado" ou "risco elevado" tem pior desempenho no teste de avaliação da capacidade aeróbia, e o conhecimento de que a prevalência de sobrepeso e obesidade em crianças tem relação com os níveis de atividadefísica ${ }^{30}$, e de que a resistência aeróbia e a aptidão física se correlacionam positivamente ${ }^{11}$, reforçam uma preocupação quase secular da comunidade científica, i.e., a associação entre a \%MG e as doenças cardiovasculares ${ }^{13}$.

Face ao exposto, parece evidente que a educação das crianças (e do cidadão em geral) sobre as consequências da obesidade, em complementaridade com o encorajamento da prática regular e sistemática de atividade física ${ }^{31}$, são medidas objetivas consagradas nas 21a , 22a , $23^{a}$ e 24a Recomendação da União Europeia ${ }^{32}$.

Em suma, o impacto da \%MG na capacidade aeróbia, reforça a importância da educação física escolar na promoção da saúde cardiorrespiratória.

$\overline{\text { Todos os autores declararam não haver qualquer potencial conflito de }}$ interesses referente a este artigo.

16. Léger LA, Mercier D, Gadoury C, Lambert J. The multistage 20-meter shuttle run test for aerobic fitness. J Sports Sci 1988;6:93-101.

17. Cooper DM, Weiler-Ravell D, Whipp BJ, Wasserman K. Aerobic Parameters of exercise as a function of body size during growth in children. J Appl Physiol 1984;56:628-34.

18. Falk B, Bar-Or O. Longitudinal changes in peak aerobic and anaerobic mechanical power of circumpubertal boys. Pediatr Exerc Sci 1993;4:36-49.

19. Silva RJ, Petroski EL. Maximum oxygen uptake and sexual maturity of children and adolescents. Motri 2008:4:13-9. 20. Rutenfranz J, Macek M, Anderson KL, Bell RD, Vávra J, Radvansky J, et al. The Relationship between changing body height and growth related changes in maximal aerobic power. Eur J Appl Physiol 1990;60:282-7. 21. Mirewald RL, Bailey DA. Maximal Aerobic Power. London: Sport Dynamic, 1986.

22. Armstrong N, Welsman J. Aerobic fitness. In: Armstrong N, Van Mechelen W, editors. Paediatric exercise science and medicine. Oxford: Oxford University Press, 2000.

23. Krahenbuhl GS, Williams TJ. Running economy: changes with age during childhood and adolescence. Med Sci Sports Exerc 1992;24:462-6.

24. Malina RM, Eisenmann JC, Cumming SP, Ribeiro B, Aroso J. Maturity-associated variation in the growth and functional capacities of youth football (soccer) players 13-15 years. Eur J Appl Physiol 2004;91:555-62.

25. Malina RM, Bouchard C, Bar-Or O. Growth, maturation, and physical activity (2 $2^{\text {nd }}$ edition). Champaign, llinois: Human Kinetics, 2004.

26. Gutin B, Yin ZN, Humphries MC, Bassali R, Le NA, Daniels S, et al. Relations of body-fatness and cardiovascular fitness to lipid profile in black and white adolescents. Pediatr Res 2005;58:78-82.

27. Lee SJ, Arslanian SA. Cardiorespiratory fitness and abdominal adiposity in youth. Eur J Clin Nutr 2007;61:561-5.

28. Ruiz JR, Rizzo NS, Hurtig-Wennlof A, Ortega FB, Warnberg J, Sjostrom M. Relations of total physical activity and intensity to fitness and fatness in children: The European Youth Heart Study. Am J Clin Nutr 2006;84:299-303.

29. Lloyd LK, Bishop PA, Walker JL, Sharp KR, Richardson MT. The influence of body size and composition on FITNESSGRAM Test Performance and the Adjustment of FITNESSGRAM Test Scores for Skinfold Thickness in Youth. Meas Phys Educ Exerc Sci 2003;7:205-26

30. Andersen LB. Physical activity in adolescentes. J Pediatr 2009;85:281-3.

31. Andersen LB, Harro M, Sardinha LB, Froberg K, Ekelund U, Brage S, Anderssen SA. Physical activity and clustered cardiovascular risk in children: a cross-sectional study (The European Youth Heart Study). Lancet 2006;368:299-304.

32. DCRP. Orientações da União Europeia para a Actividade Física. Políticas recomendadas para a Promoção da Saúde e Bem-Estar. Lisboa: Instituto do Desporto de Portugal, IP, 2009. ISBN: 978-989-8330-01-7. 